\title{
Isolating sorbicilin-producing fungi from Darband cave and evaluating the sorbicilin biomedical applications
}

\author{
Zareshahi $\mathrm{F}^{\mathbf{1}}$, Abolmaali SH${ }^{2 *}$, Darvish Alipour Astaneh $\mathrm{SH}^{1}$ and Asghari $\mathrm{A}^{3}$ \\ ${ }^{1}$ Dep. for Biotechnology, Faculty of Biotechnology, Semnan University, Iran \\ ${ }^{2}$ Dep. for Biology, Faculty of Basic Science, Semnan University, Iran \\ ${ }^{3}$ Dep. for Chemistry, Faculty of Chemistry, Semnan University, Iran
}

Zareshahi F, Abolmaali SH, Darvish Alipour Astaneh SH, Asghari A 2020 - Isolating sorbicilinproducing fungi from Darband cave and evaluating the sorbicilin biomedical applications. Studies in Fungi 5(1), 103-112, Doi 10.5943/sif/5/1/10

\begin{abstract}
To evaluate the potential of fungi from Darband cave, Semnan, Iran, for valuable antibacterial and anticancer agents, molecular screening was done against polyketides (PKS); the source for numerous diverse secondary metabolites. Fungi were isolated from soil and sludge. The antibacterial activity of the isolates was studied against indicator bacteria by well diffusion agar method, and analyzed by PCR for PKS genes. The positive strains were compared for toxicity against indicators and A549 cells. Production of antibacterial agents was investigated in 26 days followed by partial purification of the agents. Thin layer chromatography (TLC) and High Performance Liquid Chromatography (HPLC) analyses were done to reveal the nature of the toxin(s). The isolates exhibited the antimicrobial activity against Staphylococcus aureus, Bacillus cereus, and Bacillus subtilis. Three isolates selected for further studies based on the yellow culture broth, the presence of PKS, and antibacterial activity. The strains were identified as Penicillium chrysogenum using 18s rDNA analyzing. The yellow culture, $87 \%$ identity of the PKS 12/ PKS 13, the results of HPLC, and the toxic effects against certain bacteria and A549 cells confirmed the production of the sorbicillinoid in $P$. chrysogenum strains; DDFCC170 and DDFCC186. The partially-purified antibacterialanticancer agents named as AnBa170 and AnBa186 exhibited bactericide effect on S. aureus and B. cereus. These compounds killed A549 cells with an $\mathrm{IC}_{50}$ value of $0.25 \mu \mathrm{M}$ and $0.22 \mu \mathrm{M}$ respectively. AnBa170 and AnBa186 are attractive for pharmaceutical industries. Based on the literature, the cytotoxicity of sorbicillinoids against tumor cells and bacteria is related to their oxidation capacity.
\end{abstract}

Key words - Antibacterial - Penicillium chrysogenum - PKS - Sorbicillin

\section{Introduction}

Microorganisms have progressively been considered for the production of valuable drugs and chemicals (Lam 2007, Park et al. 2019). Antibiotics, antifungals, anti-virus, and anti-tumors are the most known classes of microbial by-products introduced in pharmaceutical industries (Rolain et al. 2016). However, the adaptation of microorganisms for industries as well as the retaining stability of the active molecules has been a problem. On the other hand, growing rate of cancer and antimicrobial resistance, raises greater concern to either develop or discover new drugs with new features. To overcome this problem and generate industrially viable strains for bio-based active molecule production, today's metabolic engineering, genome sequencing, and synthetic biology have brought together (Chae et al. 2017, Guzmán-Chávez et al. 2018). 
Native strains can improve quantitatively and qualitatively the production of certain bioactive molecule based on naturally occurred mutations. Discovering new native resources for either known bio-compound or new molecules is performed by molecular screening against the key gene(s) in the biosynthesis pathway (Pye et al. 2017). Polyketides and non-ribosomal peptides (NRPs) synthetases are a category of the enzymes in bacteria, filamentous fungi, and plants, that biosynthesize a diverse family of bioactive molecules. Antibiotics (erythromycin A, rifamycin S, penicillin G, vancomycin and bacitracin), antifungals (amphotericin B), anti-cancer drugs (doxorubicin, epothilone), antiparasites (avermectin), cholesterol-lowering agents (lovastatin), and immunosuppressants (rapamycin, cyclosporine, and siderophores) have been reported as the most important human medicine composed by PKS and or NRPs enzymes (Fischbach 2009)

This project aimed to discover new antibiotics and or new producer strains. A cultural collection of fungi from the Darband cave (the north of Dasht Desert, Semnan, Iran) was screened against PKS genes and their antibacterial activity. Two Penicillium strains were characterized as sorbicililinoid maker. Penicillium was the first fungal species introduced for production of $\beta$-lactam antibiotic; penicillin, albeit it's potential for construction of secondary metabolites has been still investigated (Houbraken et al. 2012). The production of penicillin G, rococoumurin, melagrin, and xanthocytysin $\mathrm{X}$ in 15 different strains of Penicillium chrysogenum were documented from indoor environments (Scott et al. 2004). The marine P. chrysogenum strain was introduced as sorbicillinoid producer (Nicoletti \& Trincone 2016). More than 40 compounds were identified from the culture broth of industrial strains of Penicillium (Salo et al. 2016). Penicillium NRRL1951 is a strain specified for sorbicillinoid production. This strain does not make any other classes of $\beta$-lactam bioactive molecules.

Currently, sorbicillinoids are known as valuable pharmaceutical molecules (Harned \& Volp 2011). Sorbicillinoids are a large family of hexaketide metabolites, including more than 90 highly oxygenated molecules produced by Aspergillus, Penicillium, Streptomyces, Trichoderma and Verticillium species (Salo et al. 2016). The first sorbicillinoid was isolated from Penicillum nutatum about 60 years ago. Up to 2016, 90 sorbicillinoids were reported with certain antibacterial, antifungal, antiviral, antioxidant and anticancer activity (Meng et al. 2016). They control the cytopathic effect induced by HIV-1 and influenza virus A (H1N1) (Nicoletti \& Trincone 2016). Moreover, bisvertinol and bisvertinolone showed activity against HL-60 cells and fungi. Oxosorbicillinol and dihydrosorbicillinol were found to be toxic for Staphylococcus aureus and Bacillus subtilis (GuzmánChávez et al. 2017). Taken together, sorbicillinoids have received growing attention for biomedicine and biotechnology based industries. Therefore, in this project we considered the potential of sorbicillinoid production in Penicillium strains from the culture collection of fungi isolated from Darband cave, Mahdishahr Semnan (3544'55.7"N, 53²1'09.3"E). No study has been performed on the biome of this region up to our knowledge. Based on the literature there is a chance to obtain bioactive molecules with specific function from unknown ecosystems (Cordell 2005).

\section{Materials \& Methods}

\section{Soil sampling and isolation of fungi}

The soil samples were aseptically collected at a depth of $20 \mathrm{~cm}$ from Darband cave, Mahdishahr Semnan (3544'55.7"N, 53²1'09.3"E) in November 2016. 25 samples were immediately taken to the laboratory and stored at $4^{\circ} \mathrm{C}$. The soil samples were suspended $(1 \mathrm{~g} / 10 \mathrm{ml})$ in normal saline for $2 \mathrm{~h}$. $100 \mu \mathrm{l}$ of the samples were cultured on Yeast Extract-Peptone-Dextrose (YPD), Potato Dextrose Agar (PDA) and Sabouraud Dextrose Agar (SDA) media followed by incubation at $37^{\circ} \mathrm{C}$ and $28^{\circ} \mathrm{C}$ for 14 days. The fungi colonies were purified by successive subculturing on YPD, PDA and SDA media. The pure cultures were maintained in Dasht Desert Fungi Culture Collection, DDFCC.

\section{Screening for PKS genes}

Fungi and yeasts from DDFCC were studied for the presence of PKS (Polyketide Synthase) genes. The DNA from fungi were extracted according to the standard protocol (Al-Samarrai \& 
Schmid 2000), and further analyzed with PKSI universal degenerated primer pairs DKF; GTG CCG GTN CCR TGNGYY TC / DKR; GCG ATG GAY CCN CARCARYG (Chiu et al. 2001, Miller et al. 2012). The PCR reaction initiated with $96^{\circ} \mathrm{C}$ for $3 \mathrm{~min}, 35$ cycles of $96^{\circ} \mathrm{C}$ for $30 \mathrm{~s}, 50^{\circ} \mathrm{C}$ for $40 \mathrm{~s}$ (DKF, DKR), and ended with a final extension of $72^{\circ} \mathrm{C}$ for $10 \mathrm{~min}$. The PCR products were sequenced by Macrogen Company (South Korea) and analyzed using NCBI-BLAST services. The candidates were considered for ITS1-4 conserved DNA using universal primers (Gao et al. 2008, Smolik et al. 2010).

\section{Evaluation of antimicrobial activity}

The antimicrobial activity of the isolates was tested by agar diffusion method against 9 indicator bacteria; Escherichia coli (ATCC 25922), Shigella (sp), Staphylococcus aureus (ATCC25923), Bacillus subtilis (ATCC 12711), Bacillus cereus (PTCC1015), Proteus (sp), Pseudomonas aeruginosa (ATCC 27853) and Klebsiella pneumonia (ATCC13883). The indicator bacteria were cultured in $\mathrm{BHI}$ medium and incubated at $37^{\circ} \mathrm{C}$ for $16 \mathrm{~h}$. An equal amount of $100 \mu \mathrm{lof}$ the inoculated culture in growth phase were seeded on nutrient agar.

The fungi isolates were cultured in YPD medium, incubated at $30^{\circ} \mathrm{C}$ for $10-30$ days, and pelleted at $8000 \mathrm{rpm}$ for 10 minutes. $130 \mu 1$ of the free cell supernatants (FCS) was loaded in the 8 $\mathrm{mm}$ wells and incubated at $37^{\circ} \mathrm{C}$ for $16 \mathrm{~h}$. The halo zones were measured (Rajalakshmi \& Mahesh 2014).

\section{Kinetics of antimicrobial agent's production}

The positive strain for PKS genes; DDFCC186, DDFCC170, and DDFCC114 were subjected to a time course test. The cultures were examined for their antibacterial activity using the disk diffusion method within 26 days on a step of $48 \mathrm{~h}$.

\section{Extraction of antimicrobial agents}

The isolates DDFCC186, DDFCC170, and DDFCC114 were fermented in YPD at the optimum time. The culture media were filtered and decanted with ethyl acetate (EtOAc) $(2: 1 \mathrm{v} / \mathrm{v})$ two times for $1 \mathrm{~h}$. The organic phase containing the metabolites was separated and dried at $25^{\circ} \mathrm{C}$. The residues were dissolved in Tris buffer $(0.01 \mathrm{M})$, sterilized by filter membrane $(0.45 \mu \mathrm{m})$ and stored at $4^{\circ} \mathrm{C}$ (Hemashenpagam 2011), named as AnBa (Anti-Bacterial agent).

\section{Minimum inhibitory concentration (MIC)/Minimum bactericidal concentration (MBC)}

The antibacterial activity of the candidates was determined by minimum inhibitory concentration. The FCS and the EtOAc-extracted were tested against B. subtilis, B. cereus and $S$. aureus. $150 \mu \mathrm{l}$ of FCS and or EtOAc-extracted were serially diluted and loaded in 96 wells plate containing $150 \mu \mathrm{l}$ nutrient broth. The indicators cultures $(0.5 \mathrm{McFarland}, 5 \mu \mathrm{l})$ were seeded in the wells. The inhibitory concentration (MIC) and minimum bactericidal concentration (MBC) were calculated in terms of volumetric volume $(\mu \mathrm{l} / \mathrm{ml})$ after $16-18 \mathrm{~h}$ at $37^{\circ} \mathrm{C}$ (Corral et al. 2018).

\section{Antibacterial activity in comparison to antibiotics and hemolytic activity}

The antibacterial activity of DDFCC186, DDFCC114, and DDFCC170 against indicator bacteria (B. subtilis, B. cereus and S. aureus) was compared with antibiotics. The indicators with 0.5 McFarland were cultured on nutrient agar. The antibiotic discs of erythromycin (15 mg), chloramphenicol (30 mg), tetracycline (30 mg), ampicillin (10 mg), amoxicillin (25 mg), and gentamicin $(10 \mathrm{mg})$ were placed on the medium where $100 \mu \mathrm{l}$ of FCS and EtOAc-extracted loaded in the wells, for $24 \mathrm{~h}$. The halo-zones were measured after $24 \mathrm{~h}$ incubation at $30^{\circ} \mathrm{C}$. DDFCC186, DDFCC114, and DDFCC170 were cultured on the sheep blood agar for $18 \mathrm{~h}$ at $37^{\circ} \mathrm{C}$ to evaluate

\section{Chemical analyses}

Thin-layer chromatography (TLC) was performed on silica gel 60. Rf values were measured applying $10 \% \mathrm{MeOH}$ in $\mathrm{CH}_{2} \mathrm{Cl}_{2}$ (Maskey et al. 2005). 
A Knauer Smartline HPLC instrument (Berlin, Germany) equipped with a quaternary HPLC pump, a UV-VIS detector (D-14163 model), and a C18 Eurospher-100 (5 $\mu \mathrm{m}$ particle, $250 \mathrm{~mm} \times 4.6$ $\mathrm{mm})$. Samples and standard solution were filtered by hydrophilic PTFE membrane $(0.45 \mu \mathrm{m})$ and injected in a volume of $20 \mu \mathrm{L}$. The chromatography data were processed using ChromGate software (version 3.1). The flow rate of $1 \mathrm{ml} / \mathrm{min}$ was applied for column elution and peaks monitored at 227 nm.

The mobile phase was a gradient of acetonitrile: $0.02 \%$ acidic water 90:10 to 25:75 in 15 min, followed by $25: 75$ back to $80: 20$ in $40 \mathrm{~min}$, then to acetonitrile in ( $45-50$ ) min. The method was ended by acetonitrile: $0.02 \%$ acidic water $10: 90$ to $55 \mathrm{~min}$ and maintained for $60 \mathrm{~min}$. The monitoring wavelength was $227 \mathrm{~nm}$. The compounds were analyzed on the retention time and chromatogram matching with the standard.

\section{Cell viability assay}

The cell line A549 (human lung carcinoma) was grown in DMEM/F12 medium (Biowest) supplemented with $10 \%$ fetal bovine serum (FBS-Biosera). Cells were maintained in humidified, $5 \%$ $\mathrm{CO}_{2}$ atmosphere at $37^{\circ} \mathrm{C}$. The cytotoxic potential of the AnBa170, AnBa186, and AnBa114 was evaluated using MTT assay. Cells $\left(2 \times 10^{4}\right.$ cells/well $)$ were seeded in the supplemented medium in 96well culture plates and were incubated in a humidified atmosphere with $5 \% \mathrm{CO}_{2}, 37^{\circ} \mathrm{C}$, for $24 \mathrm{~h}$. The medium was replaced with fresh medium supplemented with AnBa170, AnBa186, and AnBa114, in a concentration of $1 / 2$ and standard sorbicillin at $80,40,20 \mu \mathrm{M}$, followed by incubating at $5 \% \mathrm{CO}_{2}$, $37^{\circ} \mathrm{C}$, for $24 \mathrm{~h}$. To avoid the possible oxidative activity of the sorbicillin compound, the wells gently washed with $200 \mu \mathrm{l}$ of PBS (Phosphate buffer saline). Every well was subjected to $20 \mu \mathrm{L}$ of MTT in water $\left(5 \mathrm{mg} / \mathrm{ml}\right.$ ) and incubated again for $4 \mathrm{~h}$ at $5 \% \mathrm{CO}_{2}, 37^{\circ} \mathrm{C}$. Then, $100 \mu \mathrm{L}$ of dimethyl sulfoxide (DMSO) was added to dissolve the formazan crystals. The spectrophotometer analysis was done at $570 \mathrm{~nm}$ using a scanning microplate reader.

\section{Statistical analysis}

The data were tested for normality by SPSS version 22 and were then analyzed for the traits using analysis of variance and Duncan's multiple range test $(\mathrm{P}<0.05)$.

\section{Results}

\section{Soil sampling and isolation of fungi}

A total of 100 fungi were isolated from soil and sludge samples in Darband cave. The screening was performed using three media of YPD, PDA and SDA at $28^{\circ} \mathrm{C}$ and $37^{\circ} \mathrm{C} .58 .4 \%$ of the isolates were obtained at $28^{\circ} \mathrm{C}$, and $41.67 \%$ grown at $37^{\circ} \mathrm{C}$. Of the isolates, $49.1 \%$ were grown on YPD agar, 48.3\% on PDA and $1.6 \%$ on SDA medium. The antimicrobial activity of the FCS was investigated against indicator bacteria by the agar diffusion method. 26 isolates showed antimicrobial activity. The FCS from isolates did not show any effect on P. aeruginosa and E. coli, while were effective against $S$. aureus (16 isolates), B. cereus (11 isolates), B. subtilis (5 isolates) and Proteus (1 isolate). Surprisingly, three isolates were found with a distinguishable-yellow culture medium.

\section{Molecular screening against PKS genes}

The PKS gene amplicons were obtained with DKF /DKR primer pairs, respectively. The expected fragments of 700-800 bp for PKS were detected in 22 isolates.

\section{Evaluation of the antimicrobial activity of the isolates}

Fig. 1 shows the antibacterial activity of some isolates against the indicators. The existence of PKS genes in companion with the largest antibacterial spectrum made DDFCC170 as the candidate fungi for further analysis. DDFCC170 inhibited the three indicators, B. cereus, B. subtilis, and S. aureus. The growth medium for DDFCC170 obviously differed by the yellow color which probably given by carotenoid pigment. Based on the result from the agar diffusion method, producing of 
sorbicillin was supposed. The antibacterial spectrum of DDFCC186 was similar to DDFCC170 as appeared in Fig. 1

The DDFCC114 was examined because of specific antibacterial activity against $S$. aureus. In the current study, three Penicillium (sp) isolates making the culture medium yellow were detected.

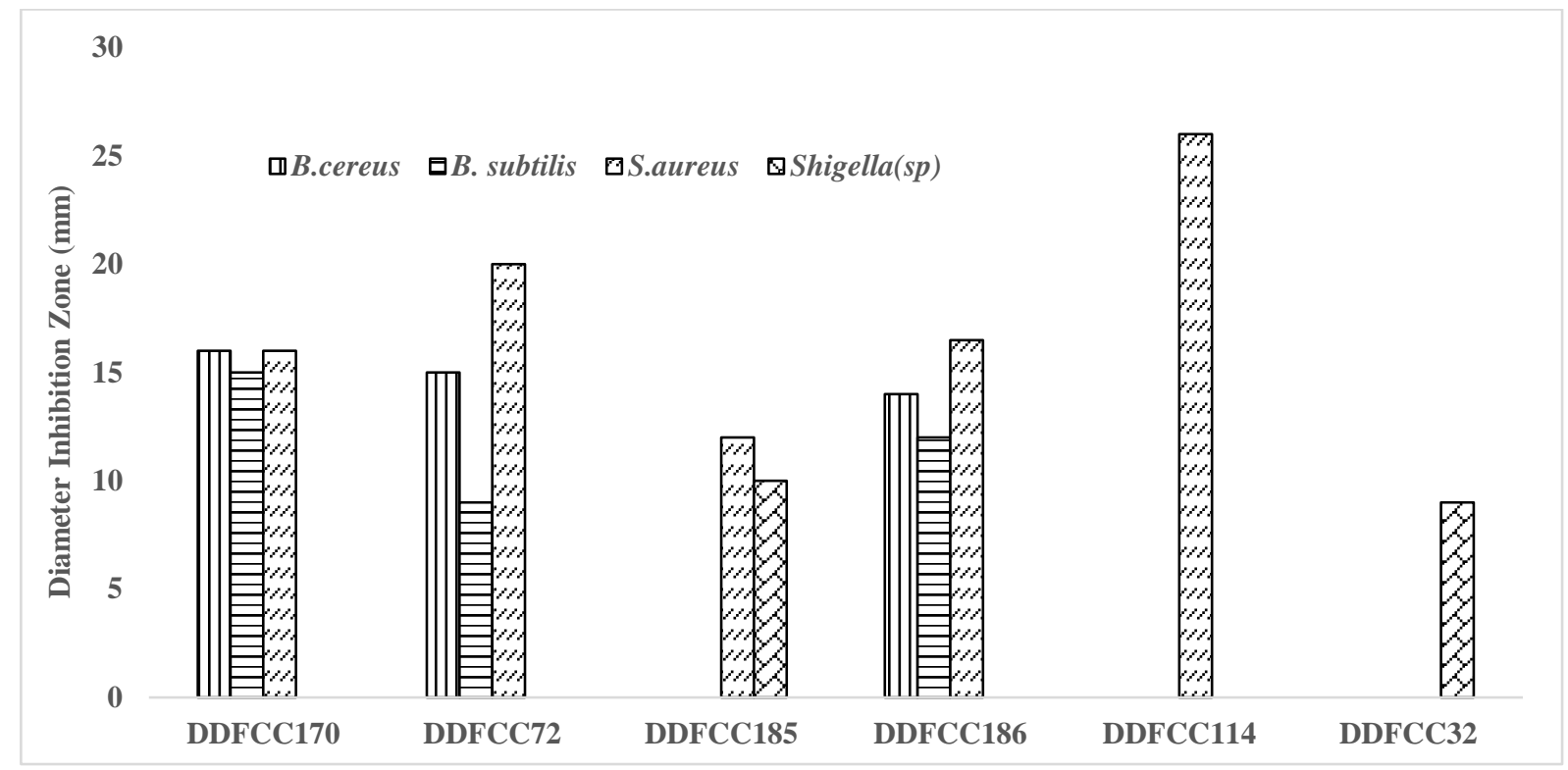

Fig. 1 - Antimicrobial activity of the FCS against indicator bacteria by agar diffusion method.

\section{Molecular identification}

The blue to blue-green conidia, yellow pigment, morphology and microscopic features and molecular identification of DDFCC170, DDFCC186, and DDFCC114 confirmed that they are $P$. chrysogenum The blastn alignment of the 700 ITS1-ITS4 bp DNA fragments showed 99\% similarity to $P$. chrysogenum for all the three strains.

\section{Kinetics of antimicrobial agent production}

The production of antimicrobial agents was assayed against indicators by disk diffusion agar for 26 days (Fig. 2). Production of AnBa186 was on the $13^{\text {th }}$ day, which reached the highest level on the $17^{\text {th }}$ day. AnBa170 was obtained on the $15^{\text {th }}$ day exhibited the largest inhibition zone $(35 \mathrm{~mm})$ against $S$. aureus. The antimicrobial compound of the DDFCC 114, AnBa114, produced within $15^{\text {th }}$ days followed by an obvious reduction (Fig. 2).

\section{MIC/MBC evaluation}

FCS of DDFCC170, DDFCC186, and DDFCC114 were extracted by EtOAc and the residue dissolved in Tris buffer. The extracts were evaluated for their antibacterial activity in comparison to FCS. The results showed that the EtOAc-extracts were more effective than the FCS against B. cereus and $S$. aureus.

AnBa170, AnBa 186 showed the highest antibacterial activity against $S$. aureus (31.2 and 62.5 $\mu \mathrm{l} / \mathrm{ml})$ and B. cereus $(62.5 \mu \mathrm{l} / \mathrm{ml})$ and DDFCC114 against $S$. aureus with the MBC value of 62.5 $\mu \mathrm{l} / \mathrm{ml}$.

\section{The antibiogram and hemolytic activity assay}

The antibiogram assay revealed that the FCS of DDFCC186 was a weak antibacterial in comparison to all the tested antibiotics. The EtOAc-purified and FCS of DDFCC170 with a diameter of $37 \mathrm{~mm}$ and $32 \mathrm{~mm}$ were the strong killers for S. aureus (Fig. 3). The AnBa114 with a halo diameter of $35.5 \mathrm{~mm}$ specifically inhibited $S$. aureus. None of the FCS and EtOAc-extraction from 
DDFCC170, DDFCC186, and DDFCC114 showed hemolytic activity, indicating that they do not destruct cells.

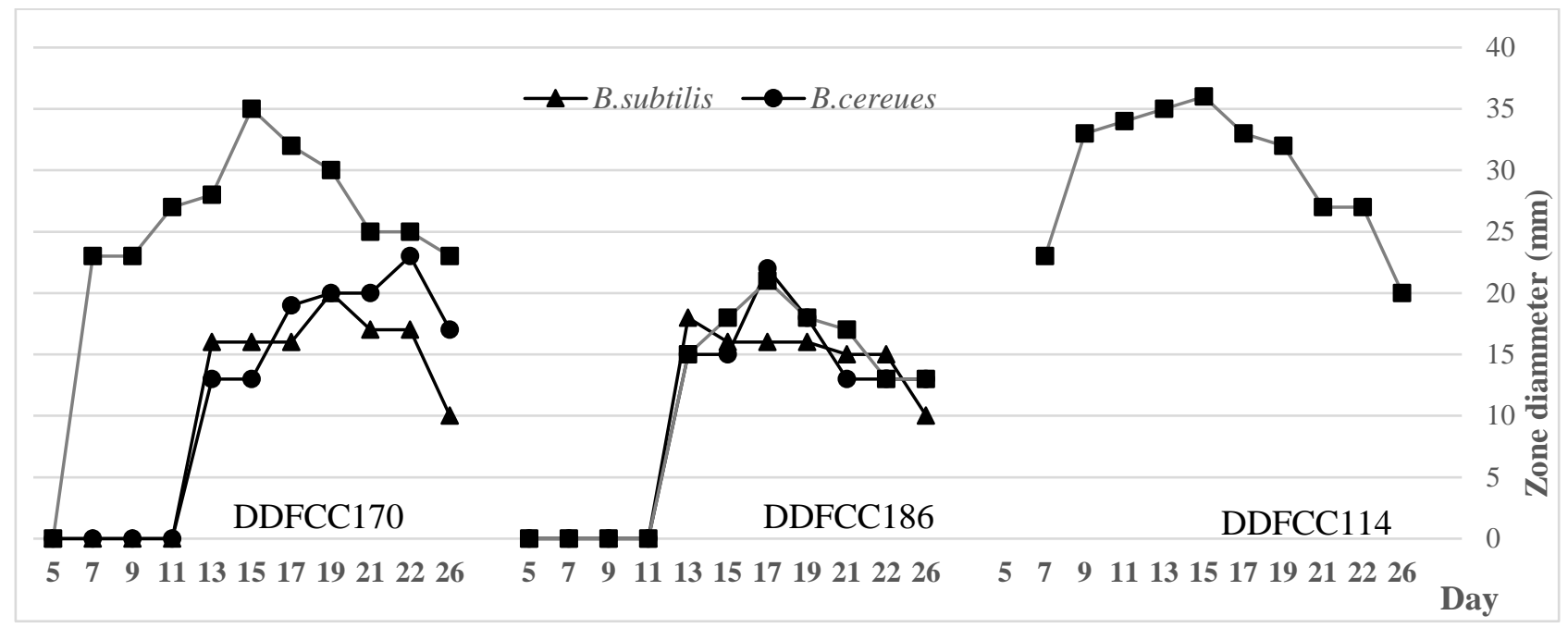

Fig. 2 - The production of antimicrobials agents assayed against indicators for 26 days

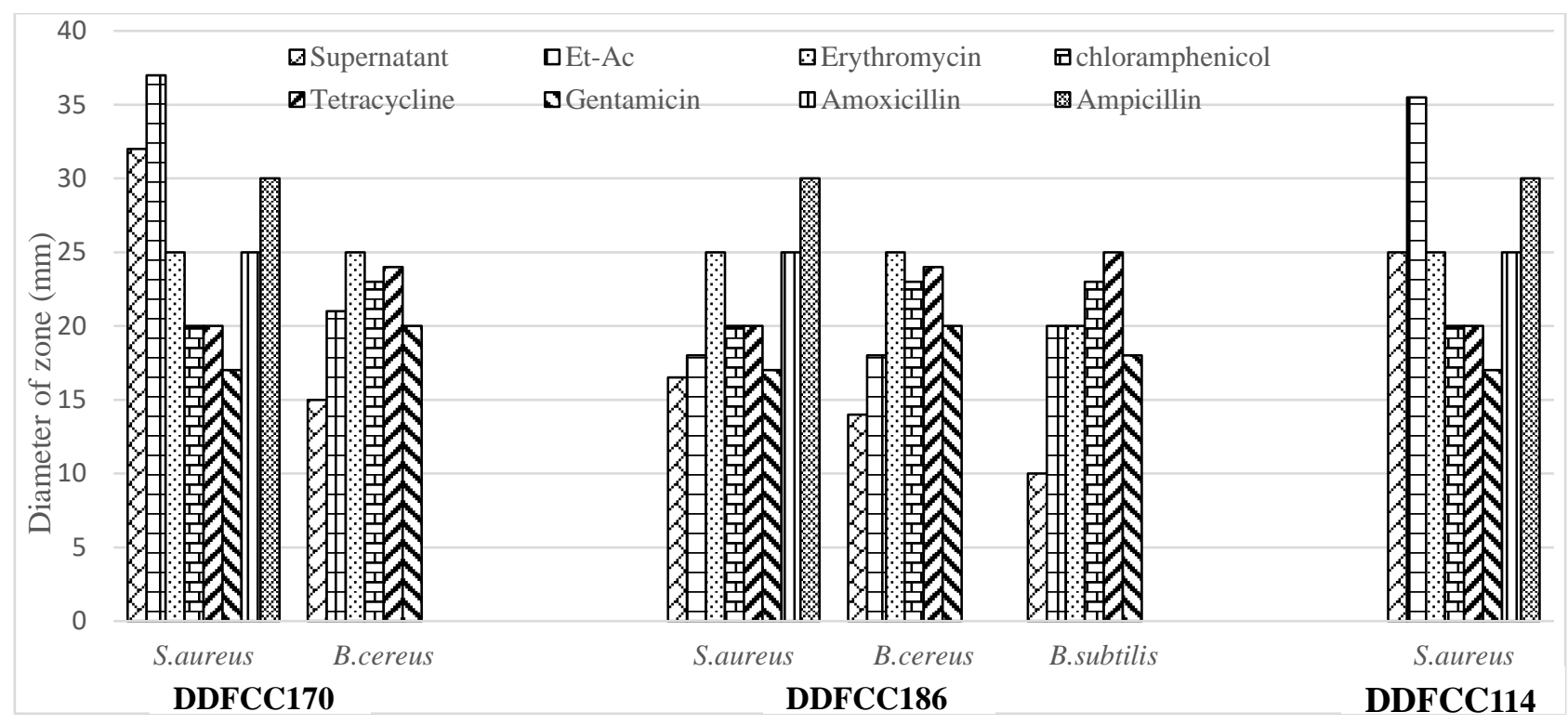

Fig. 3 - Antimicrobial activity of FCS in comparison to the antimicrobial effect of known antibiotics

\section{Chemical analyses}

AnBa170 and AnBa186 were analyzed by TLC. The bands appeared with exposing to UV in the range of $254 \mathrm{~nm}$. The band with the Rf of 0.9 value was detected for AnBa170 and AnBa186 as well as for sorbicillin.

The standard chromatogram of sorbicillin was measured using HPLC method (Fig. 4). The chromatogram of standard sorbicillin at $227 \mathrm{~nm}$ showed a peak (41.8 min) that were identified in the chromatogram of AnBa170 as retention time $(\mathrm{Rt})=41.3 \mathrm{~min}$. The peak was similarly detected in AnBa186 (41.6 min). The peaks were confirmed by the alignment of sorbicillin, AnBa170 and AnaBa 186 chromatograms (Fig. 4). At the same retention time, no peak was detected for AnBa114.

The HPLC and TLC analyses of the DDFCC 170 and DDFCC 186 partially- purified bioactive molecules proved the existence of sorbicillinoid molecules. The HPLC chromatogram of each extract showed the peak similar to the standard of sorbicillin. 


\section{Cell viability assay}

The inhibitory effects of IC50 values are presented in Fig. 5. AnBa170 showed prominent cytotoxic activity against A549 cell line with a value for IC50 as $0.25 \mu \mathrm{M}$ in $24 \mathrm{~h}$. Same experiment with AnBa186 resulted in IC50 $=0.22 \mu \mathrm{M}$ in $24 \mathrm{~h}$. The standard of sorbicillin was used as a positive control showing a strong cytotoxic effect on A549 cells.

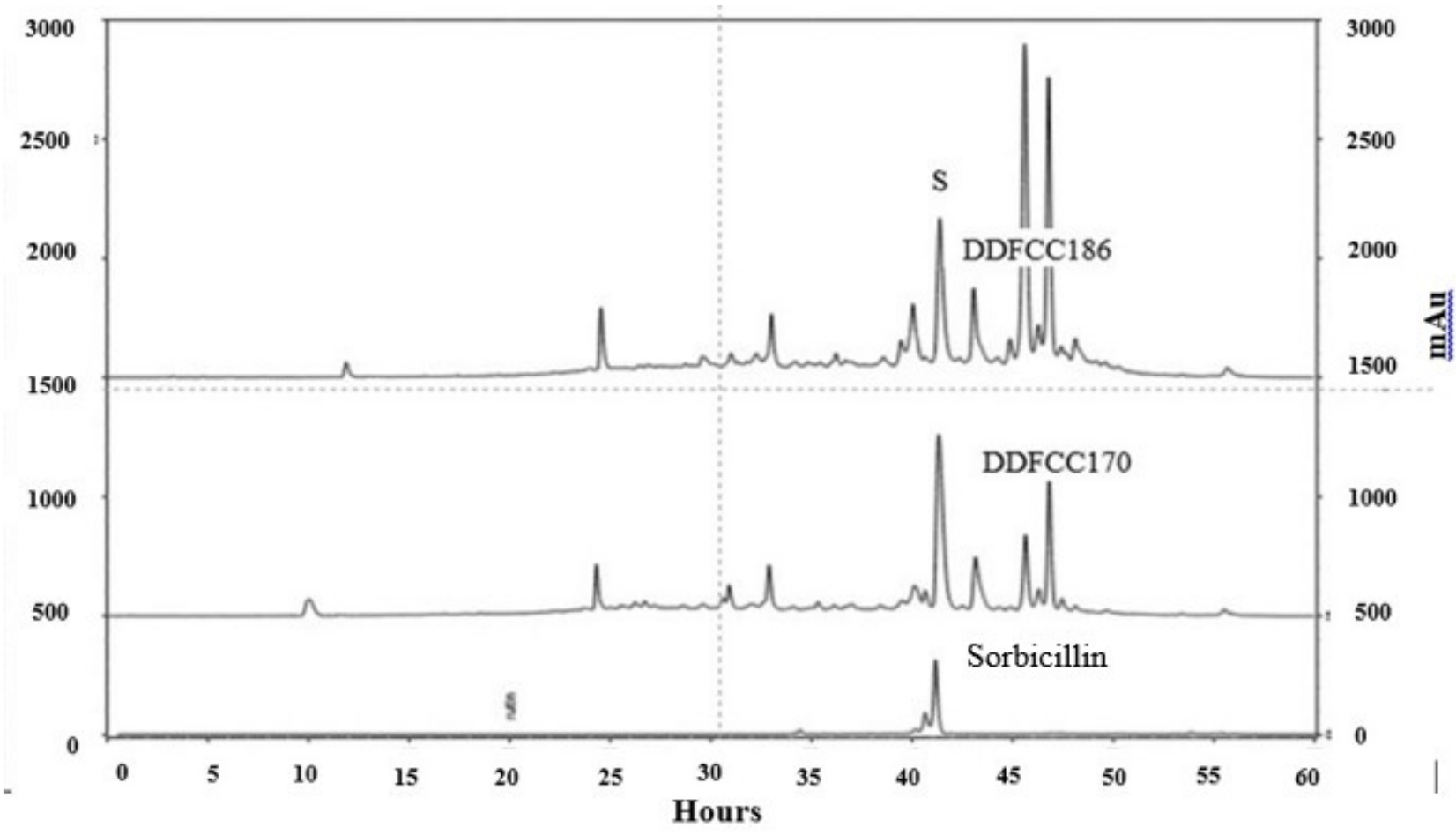

Fig. 4 - The standard chromatogram of sorbicillin in comparison to the chromatogram of AnaBa 170 and AnaBa186. The reference peak corresponding to standard sorbicillin represented by "S"

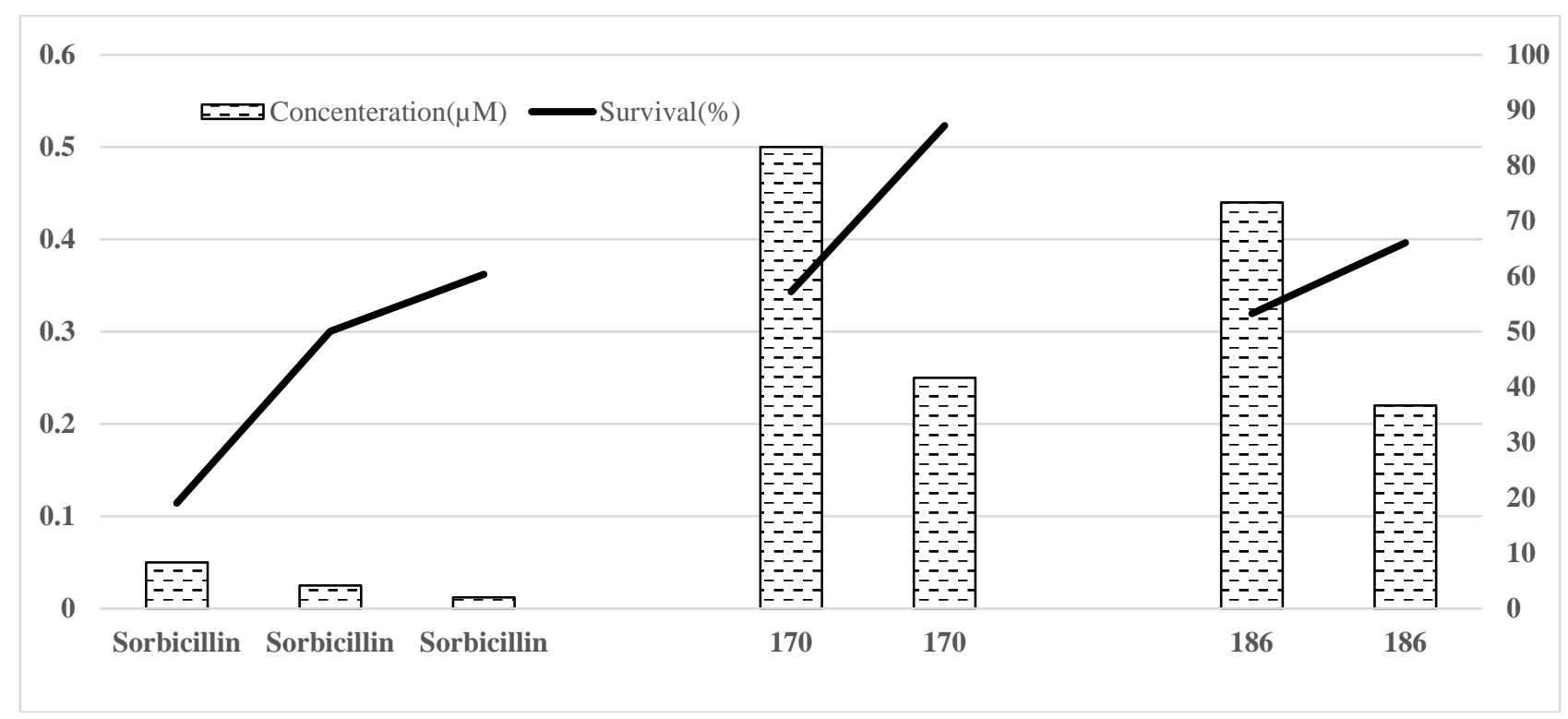

Fig. 5 - Cytotoxic effects of sorbicillin, AnaBa 170 and AnBa186 on A549 cell

\section{Discussion}

In this study, the fungal isolates from Darband cave were screened for a distinguished antibacterial activity. Among the isolates, three $P$. chrysogenum strains were introduced here with the notable antibacterial activities against $S$. aureus, $B$. subtilis, and $B$. cereus. Their yellow culture 
media made the isolates as much as interesting to be further chemically and microbiologically analyzed.

Two strains out of three, P. chrysogenum DDFCC170 and P. chrysogenum DDFCC186, showed no effect on the Gram-negative bacteria (lipopolysaccharide membrane) suggesting the hydrophilic properties for the antimicrobial agents from DDFCC170 and DDFCC186. The indicators S. aureus, B. subtilis, and B. cereus were similarly inhibited by DDFCC170 and DDFCC186 indicating the existence of some other antibacterial agents excluding penicillin derivatives. To obtain the bio-active molecules, as the polarity of the antimicrobial agent was unknown, EtOAc was used to separate both the polar and nonpolar compounds from the aqueous phase. EtOAc due to its chemical and biological properties, has a moderate polarity and minimal toxicity in the extraction of many biological and polar compounds (Altemimi et al. 2017). Ghanbari et al., partially-purified the FCS of four Penicillium isolates with EtOAc and examined the presence of bioactive compounds by GC-MS (Ghanbari et al. 2014). EtOAc-purified extraction showed optimum antimicrobial activity in the investigation done by Luca et al. (2019).

Considering the cytotoxicity effects beside yellow color in culture media, and literature (Guzmán-Chávez et al. 2017), DDFCC 170 was a candidate for production of sorbicillinoid compounds.

Sorbicillinoids are bioactive molecules with a variety of bio-features effects and growing attention for biomedicine and biotechnology based industries. The first sorbicillinoid known from Penicillium notatum identified as a contaminant in the production of penicillin, the molecules are biosynthesized by the oxidative dimerization of a hexaketide molecule (Salo et al. 2016), therefore, molecular screening based on PKS genes was a useful method (Mohanty et al. 2016).

The result of sequencing for PKS I gene was evaluated for DDFCC170 against NCBI genebank. The blastn for DDFCC170 showed a similarity of 87\% to PKS12/PKS13 (polyketide synthase) genes in $P$. chrysogenum leading to $72 \%$ identity at the amino acid level (blastx).

The putative sorbicillinoid gene cluster of industrial $P$. chrysogenum strains include two PKS genes; sorA, Pc21 g05080, and sorB, Pc21 g05070 (Salo et al. 2016). The molecular analyses of the 760 bp PKS gene fragment from DDFCC170 revealed $87 \%$ identity to sorA. Consequently, DDFCC170 was considered as a candidate for production of sorbicilinoids.

Despite of Trichoderma is known as the industrial sorbicillinoid producer, currently the marine $P$. chrysogenum strain was introduced with the active sorbicillinoid biosynthesis pathway (Li et al. 2018). Based on the literature, producing the valuable fungi secondary metabolites; sorbicillinoid compounds in these three strains was assumed (Guo et al. 2013). Unlike the improved strain, Wisconsin, producing sorbicillinoids has been shown in the native isolates of $P$. chrysogenum (Guzmán-Chávez et al. 2017). Therefore, it seems that the biosynthesis pathway for penicillin and sorbicilin are not switched on at the same strain.

The HPLC and TLC analyses of the DDFCC 170 and 186 partially-purified bioactive molecules proved the existence of sorbicillinoid molecules. The HPLC chromatogram of each extract showed the peak similar to the standard of sorbicillin. The antibacterial activity of sorbicillinoid compounds against $B$. cereus, B. subtilis, $S$. aureus was shown previously although, it has been known as an anticancer drug (Maskey et al. 2005).

DDFCC114 with the lowest intensity of yellow color in the media harbored the most efficient antibacterial agent only against $S$. aureus. None of the other indicators were limited subjecting to the antibacterial agent from DDFCC114. Therefore, DDFCC114 considered as a penicillin derivative producer via the known metabolic pathway in P. chrysogenum (Viggiano et al. 2018). Harned et al. reviewed the mechanism of cytotoxic activity of the sorbicillinoid compounds. They documented these compounds as the antioxidant agent with a broad range of activity. In many papers, sorbicillinoids have been introduced as free radical scavenger (Harned \& Volp 2011).

\section{Conclusion}

Taken together our results supported the idea that DDFCC186, DDFCC114, and DDFCC170 are three Penicillium chrysogenum strains, which the well-known penicillin and sorbicillinoid 
biosynthesis pathways may not be activated at the same rate in every strain. Two strains DDFCC170 and DDFCC186 produce sorbicillin compound to be toxic for A549 cell line and B. subtilis, B. cereus, and S. aureus. Based on our knowledge this is the first report on terrestrial P. chrysogenum.

\section{Ethical Issues}

Whole blood was taken from healthy volunteers with informed consent. The study was conducted according to ethical principles of the Declaration of Helsinki 1964 and its later amendments and comparable ethical standards.

\section{Conflict of interest}

The authors declare that they have no conflict of interest.

\section{Acknowledgements}

The authors acknowledge the Deputy for Research and Technology, Semnan University (Semnan-Iran) for supporting this study. The HPLC analyses were done at Shahed University with a great help of Prof. Tayebeh Rajabian and M.Sc. Nosrat Rahmani.

\section{References}

Al-Samarrai T, Schmid J. 2000 - A simple method for extraction of fungal genomic DNA. Letters in Applied Microbiology 30, 53-56.

Altemimi A, Lakhssassi N, Baharlouei A, Watson D, Lightfoot D. 2017 - Phytochemicals: Extraction, isolation, and identification of bioactive compounds from plant extracts. Plants 6(4), 42.

Chae TU, Choi SY, Kim JW, Ko Y-S, Lee SY. 2017 - Recent advances in systems metabolic engineering tools and strategies. Current Opinion in Biotechnology 47, 67-82.

Chiu H-T, Hubbard BK, Shah AN, Eide J et al. 2001 - Molecular cloning and sequence analysis of the complestatin biosynthetic gene cluster. Proceedings of the National Academy of Sciences 98, 8548-8553.

Cordell GA. 2005 - Natural Products: Drug Discovery and Therapeutic Medicine, Lixin Zhang, Arnold L. Demain (Eds.), Humana Press, ISBN: 1-58829-383-1. Pergamon.

Corral P, Fortunato Palma E, Pietro T et al. 2018 - Identification of a Sorbicillinoid- producing Aspergillus Strain with antimicrobial activity against Staphylococcus aureus: a New Polyextremophilic Marine Fungus from Barents Sea. Marine Biotechnology 20, 502-511.

Fischbach MA. 2009 - Antibiotics from microbes: converging to kill. Current Opinion in Microbiology 12, 520-527.

Gao Z, Li B, Zheng C, Wang G. 2008 - Molecular detection of fungal communities in the Hawaiian marine sponges Suberites Zeteki and Mycale armata. Applied and Environmental Microbiology 74, 6091-6101.

Ghanbari T, Seid Mohammadkhani H, Babaeizad V. 2014 - Identification of some secondary metabolites produced by four Penicillium species. Mycologia Iranica 1,107-113.

Guo W, Peng J, Zhu T, Gu Q et al. 2013 - Sorbicillamines A-E, nitrogen-containing sorbicillinoids from the deep-sea-derived fungus Penicillium sp. F23-2. Journal of natural products 76, 21062112.

Guzmán-Chávez F, Zwahlen RD, Bovenberg RAL, Driessen AJM. 2018 - Engineering of the Filamentous Fungus Penicillium chrysogenum as Cell Factory for Natural Products. Frontiers in Microbiology 9. doi: 10.3389/fmicb.2018.02768

Guzmán-Chávez F, Salo O, Nygård Y, Lankhorst PP et al. 2017 - Mechanism and regulation of sorbicillin biosynthesis by Penicillium chrysogenum. Microbial biotechnology 10, 958-968.

Harned AM, Volp KA. 2011 - The sorbicillinoid family of natural products: Isolation, biosynthesis, and synthetic studies. Natural Product Reports 28,1790-1810. 
Hemashenpagam N. 2011 - Purification of secondary metabolites from soil Actinomycetes. International Journal of Microbiology Research 3(3),148-156.

Houbraken J, Frisvad JC, Seifert K, Overy DP et al. 2012 - New penicillin-producing Penicillium species and an overview of section Chrysogena. Persoonia: Molecular Phylogeny and Evolution of Fungi 29,78-100.

Lam KS. 2007 - New aspects of natural products in drug discovery. Trends in Microbiology 15, 279289.

Li C, Lin F, Sun W, Yuan S et al. 2018 - Constitutive hyperproduction of sorbicillinoids in Trichoderma reesei ZC121. Biotechnology for Biofuels 11, 291. doi: 10.1186/s13068-018-1296-4

Luca SV, Miron A, Ignatova S, Skalicka-Woźniak K. 2019 - An overview of the two-phase solvent systems used in the countercurrent separation of phenylethanoid glycosides and iridoids and their biological relevance. Phytochemistry Reviews,1-27.

Maskey RP, Grün-Wollny I, Laatsch H. 2005 - Sorbicillin Analogues and Related Dimeric Compounds from Penicillium notatum. Journal of natural products 68, 865-870.

Meng J, Xiaohan W, Dan X, Xiaoxiang F et al. 2016 - Sorbicillinoids from fungi and their bioactivities. Molecules 21(6), 715.

Miller KI, Qing C, Sze DMY, Neilan BA. 2012 - Investigation of the biosynthetic potential of endophytes in traditional Chinese anticancer herbs. PLoS One 7, e35953.

Mohanty D, Khater S, Anand S. 2016 - In silico methods for linking genes and secondary metabolites: The way forward. Synthetic and Systems Biotechnology1(2), 80-88.

Nicoletti R, Trincone A. 2016 - Bioactive compounds produced by strains of Penicillium and Talaromyces of marine origin. Marine drugs 14(2), 37.

Park SR, Yeo Joon Y, Janette VP, Mariamawit AY et al. 2019 - A review of the microbial production of bioactive natural products and biologics. Frontiers in Microbiology 10, 1404. doi: 10.3389/fmicb.2019.01404.

Pye CR, Bertin MJ, Lokey RS, Gerwick WH, Linington RG. 2017 - Retrospective analysis of natural products provides insights for future discovery trends. Proceedings of the National Academy of Sciences 114, 5601-5606.

Rajalakshmi S, Mahesh N. 2014 - Production and characterization of bioactive metabolites isolated from Aspergillus terreus in rhizosphere soil of medicinal plants. International Journal of Current Microbiology and Applied Sciences 3,784-798.

Rolain J-M, Abat C, Jimeno M-T, Fournier P-E, Raoult D. 2016 - Do we need new antibiotics? Clinical Microbiology and Infection 22,408-415.

Salo O, Guzmán-Chávez F, Ries MI, Lankhorst PP et al. 2016 - Identification of a polyketide synthase involved in sorbicillin biosynthesis by Penicillium chrysogenum. Applied and Environmental Microbiology 82, 3971-3978.

Scott J, Untereiner WA, Wong B, Straus NA, Malloch D. 2004 - Genotypic variation in Penicillium chysogenum from indoor environments. Mycologia 96,1095-1105.

Smolik M, Andrys D, Franas A, Krupa-Malkiewicz M, Malinowska K. 2010 - Polymorphism in Syringa rDNA regions assessed by PCR technique. Dendrobiology 64, 55-64.

Viggiano A, Oleksandr S, Hazrat A, Wiktor S et al. 2018 - Pathway for the Biosynthesis of the Pigment Chrysogine by Penicillium chrysogenum. Applied and Environmental Microbiology 84,e02246-02217. 